\title{
Isolated Mesoporous Microstructures Prepared by Stress Localization-Induced Crack Manipulation
}

Sanghyuk Wooh, ${ }^{\dagger, \perp}$ Soojin Lee, ${ }^{\dagger, \perp}$ Yunchan Lee, ${ }^{\dagger, \perp}$ Ji Ho Ryu, ${ }^{\ddagger}$ Won Bo Lee, ${ }^{\ddagger}$ Hyunsik Yoon, $*, \|$ and Kookheon Char* ${ }^{\dagger}$

${ }^{\dagger}$ The National Creative Research Initiative Center for Intelligent Hybrids, The World Class University Program for Chemical Convergence for Energy and Environment, School of Chemical and Biological Engineering, Seoul National University, Seoul 08826, Republic of Korea

${ }^{\ddagger}$ School of Chemical and Biological Engineering, Seoul National University, Seoul 08826, Republic of Korea

"Department of Chemical and Biomolecular Engineering, Seoul National University of Science \& Technology, Seoul 01811, Republic of Korea

\section{Supporting Information}

ABSTRACT: Cracks observed in brittle materials are mostly regarded as defects or failures. However, they can be a valuable tool when implemented in a controlled way. Here, we introduce a strategy to control the crack propagation of mesoporous micropatterns (prisms and pyramids), which leads to the isolation of well-defined microstructures. Mesoporous micropatterns were fabricated
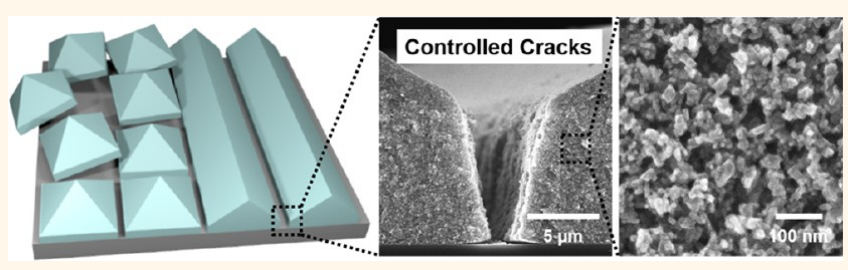
by the soft imprinting technique with wet $\mathrm{TiO}_{2}$ nanoparticle (NP) pastes, followed by sintering to remove organic components. Since the volume of the paste significantly shrinks during the sintering step, stress is localized at the edge of micropatterns, in good agreement with finite element method simulations, creating well-defined cracks and their propagation. It was demonstrated that the degree of stress localization is determined by the thickness of residual layers, NP size, and heating rate. After controlled crack propagation and delamination of microparticles from the substrates, mesoporous microwires and microparticles were successfully produced and functionalized from the isolated mesoporous prisms and pyramids. The method proposed in this study for controlled crack manipulation and delamination opens a door for straightforward and economical fabrication of well-defined mesoporous microparticles.

KEYWORDS: crack manipulation, soft lithography, stress localization, delamination, mesoporous prism(s), mesoporous pyramid(s)

$\mathrm{E}$ very material exhibits its own deformation characteristics when it is under the influence of thermal, mechanical, and/or intrinsic residual stresses. Under a compressive or a tensile stress, soft materials are spontaneously deformed into wrinkles or folds, ${ }^{1-6}$ whereas hard brittle materials are typically fractured or cracked in an effort to find a stable equilibrium. ${ }^{7-10}$ In many cases, those deformations are generated randomly and considered as defects, which are avoided to realize homogeneous and durable structures. ${ }^{11-13}$ Nevertheless, there has been much effort to exploit such deformations on various soft materials, e.g., wrinkles and folds, for valuable applications such as tunable microlenses, ${ }^{14,15}$ transistors, ${ }^{16}$ piezoelectrics, ${ }^{17}$ and photovoltaic cells. ${ }^{18,19}$ One of the deformation modes for hard materials, a "crack", is more difficult to manage than for soft materials due to its random generation and great complexity. Therefore, crack generation has been studied to understand its mechanism and to control cracking. $^{20-25}$ Recently, cracks on various hard, inorganic materials have been controlled on different length scales by using microtips ${ }^{26}$ or notches. ${ }^{27}$ In addition, the use of cracks has been well demonstrated for many scientific applications such as a multifunctional sensors ${ }^{28}$ or nanopatterning ${ }^{29}$ with facile and economically viable processes.

Depending on how we use them, it is believed that controlled cracks can be a useful tool. In this study, we propose a crack manipulation strategy on mesoporous $\mathrm{TiO}_{2}$ micropatterned structures by employing a soft imprinting technique with a wet $\mathrm{TiO}_{2}$ nanoparticle (NP) paste film, leading to the isolation of a single, well-defined structure. Previously, the soft imprinting method on wet $\mathrm{TiO}_{2} \mathrm{NP}$ paste films has been reported for the fabrication of hierarchical mesoporous $\mathrm{TiO}_{2}$ patterns. ${ }^{30,31}$ From

Received: May 8, 2016

Accepted: September 12, 2016

Published: September 12, 2016 

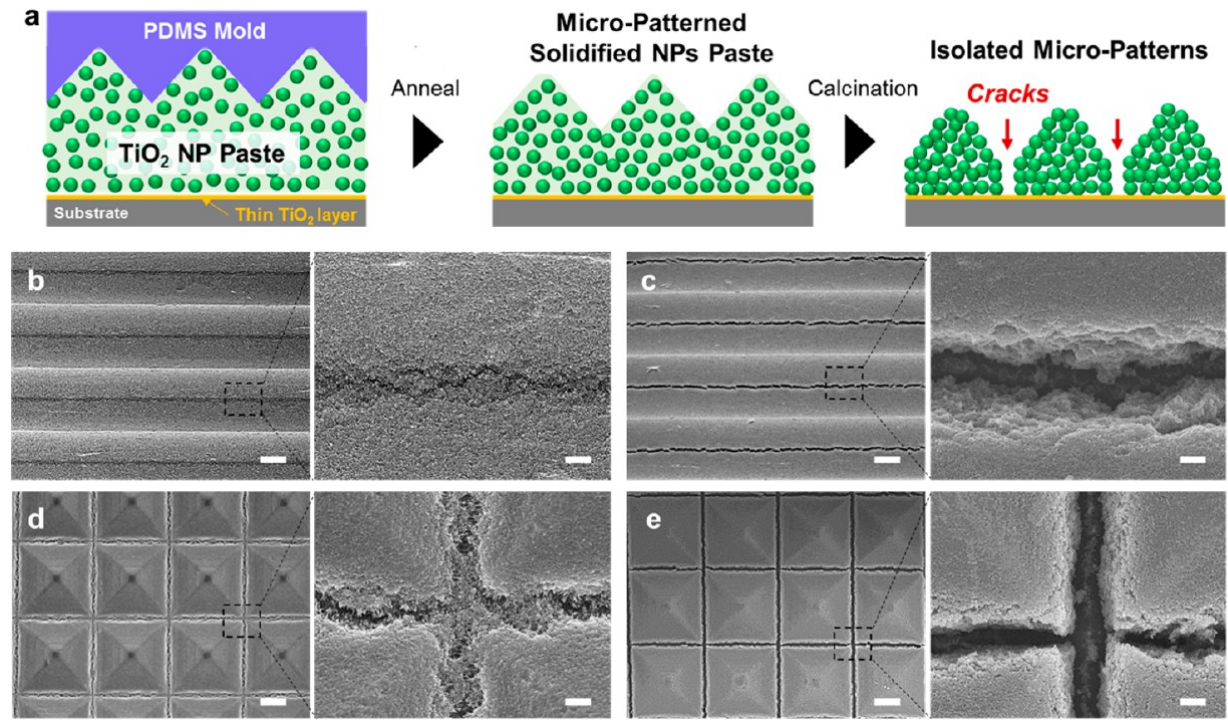

Figure 1. (a) Schematic illustration on the fabrication of isolated microstructures by crack manipulation. SEM images of (b and c) prism- and (d and e) pyramid-shaped micropatterns from wet $\mathrm{TiO}_{2} \mathrm{NP}$ pastes by soft imprinting before $\left(\mathrm{b}\right.$ and $\mathrm{d}$ ) and after $(\mathrm{c}$ and $\mathrm{e})$ sintering at $500{ }^{\circ} \mathrm{C}$ for 20 min. Right sides of $b$-e show the high magnification images of valley regions of the patterns. The scale bars in the left-side and rightside images of $b-e$ are 10 and $1 \mu \mathrm{m}$, respectively.

this method, a thin $\mathrm{TiO}_{2}$ paste film $(20-50 \mu \mathrm{m}$ thick) coated on a substrate was imprinted with micropatterned soft polydimethylsiloxane (PDMS) molds, followed by sintering at $\sim 500{ }^{\circ} \mathrm{C}$. The $\mathrm{TiO}_{2} \mathrm{NP}$ paste contained $\mathrm{TiO}_{2} \mathrm{NPs}$ mixed with solvents and organic binders. Therefore, during the sintering, all of the organics were thermally decomposed and the volume of the total body significantly shrunk. In particular, the volume shrinkage of the patterns with sharp-edged structures (e.g., prisms and pyramids) imposed local mechanical stresses at the edges. The degree of residual stress was determined by the thickness of residual layers under the patterned structure, such that cracks start to propagate when the thickness of the residual layer is above a critical thickness. In addition, the effects of NP size of $\mathrm{TiO}_{2}$ pastes as well as the heating rate on the crack mechanism were investigated. Those experimental results were all in good agreement with the previous literature studies of the effects on particle size and evaporation rate of crack propagation in drying colloidal films.

Cracks along the valleys of mesoporous $\mathrm{TiO}_{2}$ micropatterns led to the isolation of single structures. Those isolated structures could be detached from substrates when they were produced on the low adhesive substrates. For example, isolated prisms were easily detached by gentle $\mathrm{N}_{2}$ blowing, realizing mesoporous microwires which can be applied to various electrochemical applications, e.g., photocatalysts ${ }^{35,36}$ and supercapacitors. ${ }^{37}$ In the case of pyramids, the cracks formed "brownie"-like micropyramids on a substrate. Each isolated micropyramid could also be detached from the substrate by sonication and readily dispersed in solvent as single particles consisting of $\mathrm{TiO}_{2}$ NPs (i.e., mesoporous pyramidal $\mathrm{TiO}_{2}$ microparticles). For more than a decade, mesoporous particles have received great attention due to their functionalities and good processability. ${ }^{38-40}$ Most mesoporous particles have been prepared by solution processes in which it is hard to control porosity and difficult to tune size uniformly. However, this preparation concept of mesoporous microwires and microparticles based on crack manipulation is straightforward and porosity tunable and makes it possible to realize uniform size with one imprinting step. From this standpoint, we believe that proper crack control in mesoporous microstructures will prompt diverse scientific research with this simple and economic process.

\section{RESULTS AND DISCUSSION}

Figure 1a shows the schematic on the fabrication of isolated mesoporous structures by crack manipulation. Mesoporous micropatterns were fabricated by the soft imprinting with an asprepared micropatterned polydimethylsiloxane (PDMS) mold in contact with a commercial wet $\mathrm{TiO}_{2} \mathrm{NP}$ paste (18NR-T, Dyesol) film. Before the wet $\mathrm{TiO}_{2} \mathrm{NP}$ paste was coated, a thin $\mathrm{TiO}_{2}$ film $(\sim 100 \mathrm{~nm})$ was first prepared on a substrate to secure better adhesion between the substrate and mesoporous $\mathrm{TiO}_{2}$ patterns. A doctor blade coating method was employed to coat the $\mathrm{TiO}_{2}$ paste followed by imprinting with a PDMS mold replicated from a master in a prism or pyramid shape (Figure S1) and annealing at $60{ }^{\circ} \mathrm{C}$ to remove most of the residual solvent with pattern shape retained. The solidified micropatterned paste was further sintered at $500{ }^{\circ} \mathrm{C}$ to remove all of the organics remaining in the paste. Then, the mesoporous microstructure assembled from $\mathrm{TiO}_{2}$ NPs was finally obtained. During the sintering steps, thermogravimetric analysis and scanning electron microscope (SEM) showed that $\sim 30 \%$ of weight and $\sim 24 \%$ of volume contraction took place (Figure S2). This volume shrinkage obviously causes internal stresses; in particular, for the cases of inclined shape patterns such as prisms and pyramids, the internal residual stresses are concentrated at the sharp edges in contact with $\mathrm{TiO}_{2} \mathrm{NP}$ paste films which, in turn, directly influence the crack generation. Parts $b$ and $d$ of Figure 1 show the SEM images of patterned $\mathrm{TiO}_{2} \mathrm{NP}$ pastes after the first annealing step at 60 ${ }^{\circ} \mathrm{C}$, which were imprinted by prism and pyramid micropatterns (25 and $30 \mu \mathrm{m}$ width for prism and pyramid, respectively, with $45^{\circ}$ inclination). Before sintering, imprinted patterns had crack initiation tips generated at the sharp edges, but an individual patterned structure was still connected (see high magnification images of Figure $1 \mathrm{~b}, \mathrm{~d}$ on the right-hand side). It was observed that cracks were readily propagated during the sintering step by 

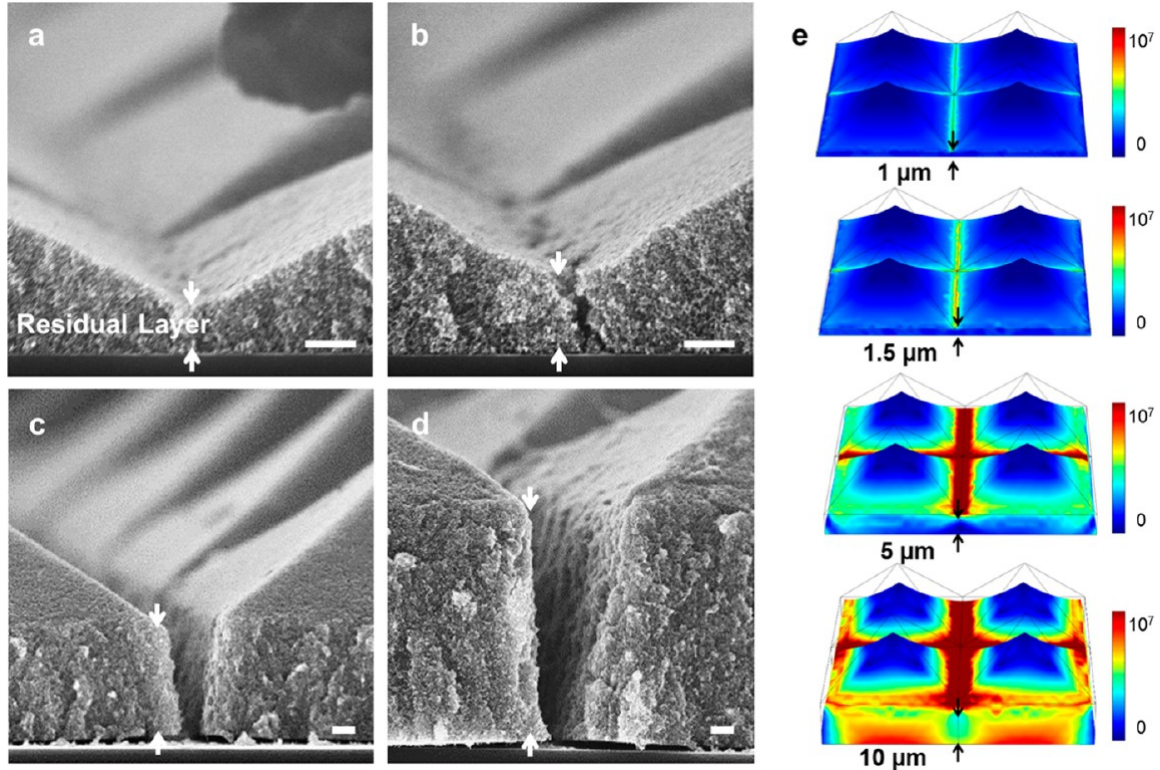

Figure 2. Cross-sectional SEM images of mesoporous micropyramid patterns with different thicknesses of residual layers: (a) 1, (b) 1.5, (c) 5, (d) $10 \mu \mathrm{m}$. The scale bars indicate $1 \mu \mathrm{m}$. (e) Stress distribution, simulated by FEM, on patterned pyramids with different residual layer thickness as a result of volume shrinkage of the pastes during the sintering step.
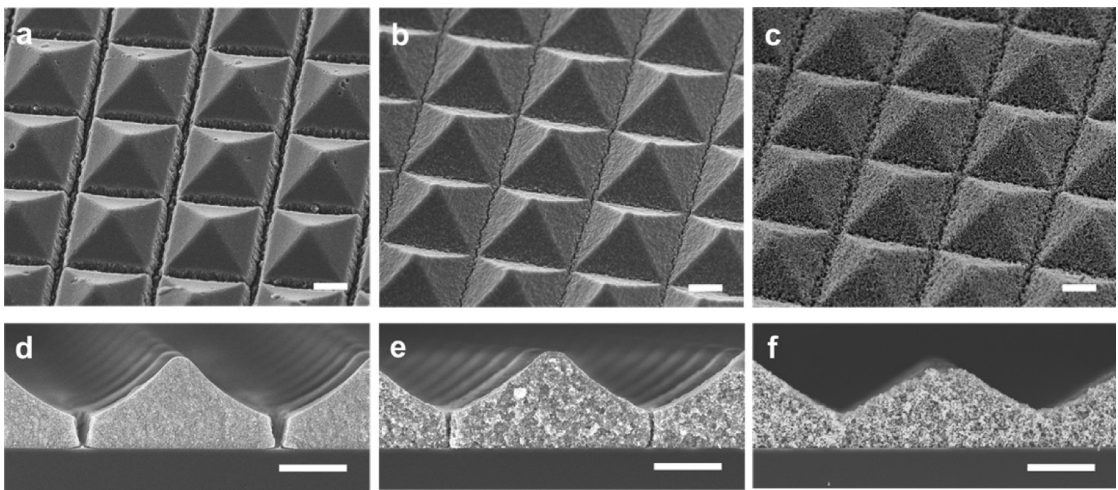

Figure 3. Top and cross-sectional SEM images of mesoporous $\mathrm{TiO}_{2}$ pyramids with different sizes of NPs, 20 nm in diameter (a and d), 1:1 mixture of 20 and $250 \mathrm{~nm}$ (b and $e$ ), and $250 \mathrm{~nm}$ in diameter ( $c$ and $\mathrm{f}$ ). After the pyramid-patterned pastes containing $20 \mathrm{~nm}$ NPs were sintered, cracks were clearly visible. However, the crack opening is not pronounced in the case of a mixture of two different NP size, and no cracks were even initiated in the pyramid patterns containing $250 \mathrm{~nm}$ NPs. The scale bars indicate $10 \mu \mathrm{m}$.

localized stress derived from volume shrinkage of the paste, and well-ordered cracks with $1-1.5 \mu \mathrm{m}$ width clearly appeared along the valleys of both prism and pyramid patterns (Figure $1 c, e)$. We also investigated the effect of sintering temperature (Figure S3). Patterned samples were heated to different temperatures, 150,250 , and $350{ }^{\circ} \mathrm{C}$, and maintained for $2 \mathrm{~h}$. We note that micropatterns were connected when they were sintered at $150{ }^{\circ} \mathrm{C}$ (Figure S3a-c) while cracks started to propagate by the degradation of organics over $250{ }^{\circ} \mathrm{C}$ (Figure $\mathrm{S} 3 \mathrm{~d}-\mathrm{f})$, and the patterns were isolated from each other. However, the color of the film was still yellowish (see Figure S3d), indicating that the organic binder was not fully decomposed at $250{ }^{\circ} \mathrm{C}$. When we increased the sintering temperature to $>350{ }^{\circ} \mathrm{C}$, all of the organic components in the $\mathrm{TiO}_{2}$ pastes were decomposed and the yellowish color finally disappeared (Figure S3g). In this study, in order to guarantee the complete sintering of NPs, we sintered the samples at 500 ${ }^{\circ} \mathrm{C}$. We also assumed that the temperature was uniform across the entire microstructure. This argument is reasonable because the structures in the present study are only few microns thick with reasonably high thermal conductivity $\left(k_{\mathrm{TiO} 2}=4-12 \mathrm{~W} / \mathrm{m}\right.$. $\mathrm{K})$. When we use the criterion of Biot number $(\mathrm{Bi} \sim h L / k$ where $h$ is the convective heat transfer coefficient $(10-1000$ $\left.\mathrm{W} / \mathrm{m}^{2} \cdot \mathrm{K}\right), L$ is the characteristic length $(\sim 10 \mu \mathrm{m})$, and $k$ is the conductivity of the solid $\left.\left(k_{\mathrm{TiO} 2}=-12 \mathrm{~W} / \mathrm{m} \cdot \mathrm{K}\right)\right)$, the Bi number in our case is below unity, indicating that the $\mathrm{TiO}_{2}$ microstructures have uniform temperature distribution.

The crack location was determined by the shape of pattern molds used. In addition, we managed the crack propagation by varying the thickness of residual layers under the patterned structure, which was determined by the initial film thickness coated by the doctor blade coating. Since the stress at the pattern edge is generated by the volume shrinkage in the film thickness direction, the degree of localized stress increases with the increase in residual layer thickness. It has previously been studied that the critical strain $\left(\varepsilon_{\mathrm{c}}\right)$ required to generate a crack across a film is proportional to $(1 / h)^{0.5}$, where $h$ is the film thickness. ${ }^{41}$ Figure 2 shows the pyramid patterns with different residual layer thicknesses. When the residual layer thickness is lower than $1 \mu \mathrm{m}$, cracks do not propagate at the patterned edge 
due to insufficient localized stress (Figure 2a). However, cracks start to form at the edges of pyramid patterns with a $\sim 1.5 \mu \mathrm{m}$ thick residual layer (Figure $2 \mathrm{~b}$ ), and it was further shown that the pyramidal patterns with residual layers above $5 \mu \mathrm{m}$ produce pronounced cracks at the contacting edges (Figure $2 \mathrm{c}, \mathrm{d}$ ). From our observations, the critical cracking thickness of residual layer of pyramids is $1.4 \pm 0.2 \mu \mathrm{m}$. This effect of residual layer thickness on crack propagation was also studied with the finite element method (FEM) analysis (see the simulation discussion). The simulation results in Figure $2 \mathrm{e}$ showed that the stress was localized or concentrated at the valleys of pyramid patterns, and the stress localization in the pyramids is more pronounced with thicker residual layers. In addition, we found the same tendency of stress localization for cracks generated on prism patterns (Figure S4). We note that the delamination off the substrates occurs after cracks first start to propagate into the valleys of the patterns and finally reach the substrates, as shown in Figure 2 (for pyramids) and Figure S3 (for prisms). Note that the current FEM simulations only give insight on the stress concentration for the patterned layers. However, it is expected that the stress will dramatically change when the fracture propagates. In order to fully incorporate the fracture propagation, more elaborate simulations are needed, taking into account the stress intensity factor or the energy release rate in a geometry where the fracture propagates.

We also investigated the effect of NP size of $\mathrm{TiO}_{2}$ pastes on the crack propagation in the patterned structures. We have employed three different $\mathrm{TiO}_{2}$ pastes, $20 \mathrm{~nm}$ in diameter, 250 $\mathrm{nm}$ in diameter, and a 1:1 (w/w) mixture of each NP. After the pyramid-patterned pastes containing $20 \mathrm{~nm}$ NPs were sintered, cracks were clearly visible (Figure 3a,d). However, by increasing the average size of NPs, cracks were less opened, and no cracks were eventually observed in the pyramid patterns with $250 \mathrm{~nm}$ NPs (Figure 3c,f). These results are qualitatively in good agreement with the previous literature dealing with drying hard colloidal films, showing that the critical cracking thickness $\left(h_{\max }\right)$ scales with the radius of particles $(R)$ as $h_{\max } \propto$ $R^{3 / 2} 21,24,25$

Moreover, we could control the crack propagation by manipulating the heating rate. There have been reports on the control of crack propagation in drying colloidal films by the rate of solvent evaporation. ${ }^{7,32-34}$ Inspired by the previous reports, we performed the sintering experiments with different heating rates, $2,10,20$, and $40{ }^{\circ} \mathrm{C} / \mathrm{min}$, to clarify the effect of heating speed (which is, in turn, related to the speed of evaporating organic binders or solvents). As shown in Figure 4, higher heating rate intensifies the stress localization in the valleys of the patterns, leading to easier isolation of the patterns.

Those controlled cracks formed at the pattern edges disable the connection of micropatterned structures (i.e., isolated microprisms and micropyramids). Separate mesoporous $\mathrm{TiO}_{2}$ structures were formed by crack propagation on adhesive substrates (i.e., $\mathrm{TiO}_{2}$ adhesion promoting layers coated on $\mathrm{Si}$ wafer). However, isolated mesoporous structures detached from the substrates were obtained from the substrates such as bare silicon wafers and quartz glasses without additional $\mathrm{TiO}_{2}$ adhesion promoting layers, as clearly verified in Figure 5. Consequently, isolated prisms and pyramids were easily and completely removed from the bare substrates by either blowing air for long prism wires (Figure $5 b$ ) or sonication in ethanol for pyramidal particles (Figure $5 \mathrm{~d}$ ).

Taking full advantage of the delamination of mesoporous $\mathrm{TiO}_{2}$ patterns with low adhesion energy on bare substrates,
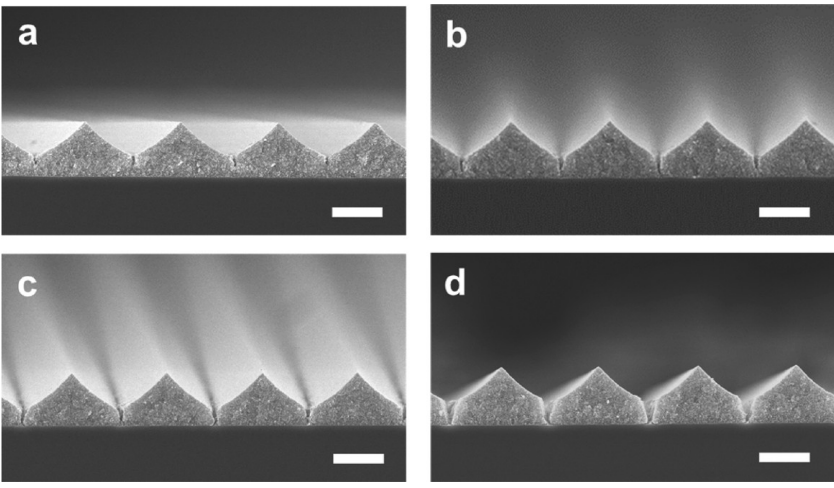

Figure 4. Cross-sectional SEM images of mesoporous $\mathrm{TiO}_{2}$ prisms heated to $500{ }^{\circ} \mathrm{C}$ with different heating rates: (a) 2, (b) 10, (c) 20, (d) $40{ }^{\circ} \mathrm{C} / \mathrm{min}$. It is noted that the increase in the heating rate intensify the stress localization in the valleys of the patterns, causing easier isolation of the patterns. The scale bars indicate 5 $\mu \mathrm{m}$.

various mesoporous microwires with different aspect ratio and microparticles can be easily prepared. To fabricate such features, cracks were initially generated and propagated in a programmed way (i.e., stress localization of patterned surfaces), followed by easy delamination of such features off the substrates with weak adhesion energy (Figure 6a). Large quantities of isolated mesoporous prisms with different aspect ratios were thus easily obtained (Figure 6b,c). In addition, prism wires with different widths $(10,25$, and $50 \mu \mathrm{m})$ were also successfully obtained (Figures S5 and S6). In addition, the isolated mesoporous $\mathrm{TiO}_{2}$ pyramids with two-dimensional (2D) cracks generated at the patterned edges were also removed from the wafer by ultrasonication for a few seconds and readily dispersed in ethanol, forming mesoporous $\mathrm{TiO}_{2}$ pyramid microparticle suspensions (Figure $6 \mathrm{~d}$ and Supporting Movie S1). Figure $6 \mathrm{~d}$ shows the remaining pyramids on a substrate after $1 \mathrm{~s}$ sonication, which has clear traces of detached pyramids. Subgram-scale mesoporous $\mathrm{TiO}_{2}$ microparticles with homogeneous size and shape were readily produced by the simple cracking and sonication process (Figure 6e). Until now, mesoporous microparticles have been mostly synthesized by solution processes in batches or microfluidics. However, this preparation method suggests the mesoporous particle preparation by a simple soft imprinting technique on the reusable substrates that requires no additional purification step and minimizes the chemical waste.

The surface area of mesoporous microparticles could be controlled by pastes containing NPs in different size (diameters: $50 \mathrm{~nm}$ and a 1:1 mixture of 20 and $250 \mathrm{~nm}$ NPs), as shown in Figure 7a,b. However, we experienced difficulty in fabricating mesoporous particles with a large NP size of $\sim 250 \mathrm{~nm}$. Due to the low cohesive interactions among big NPs, it was noted that the pyramidal structures were often destroyed during sonication (Figure S7a,c). To overcome this issue, we employed the additional $\mathrm{TiCl}_{4}$ post-treatment after preparing separate mesoporous pyramids to enhance the formation of NP network. ${ }^{42}$ This additional treatment is believed to create thin $\mathrm{TiO}_{2}$ coating layers among large NPs, increasing the hardness of the structure ${ }^{43}$ and allowed us to easily detach the pyramidal particles containing large NPs from the substrates (Figure $7 c$ and Figure S7b,d). In addition, the volume of microparticles can be tuned by varying the residual layer thickness with smaller NPs, as shown in Figure $7 \mathrm{~d}$. 


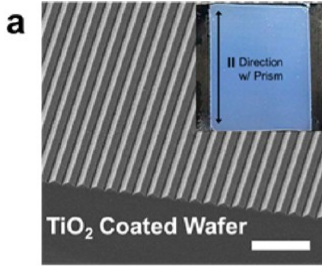

b

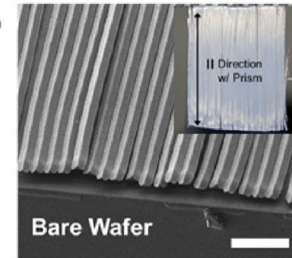

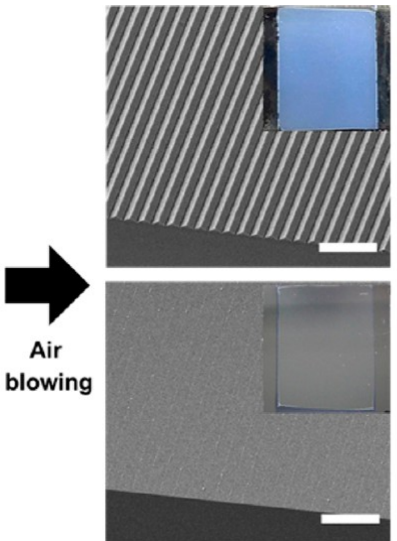
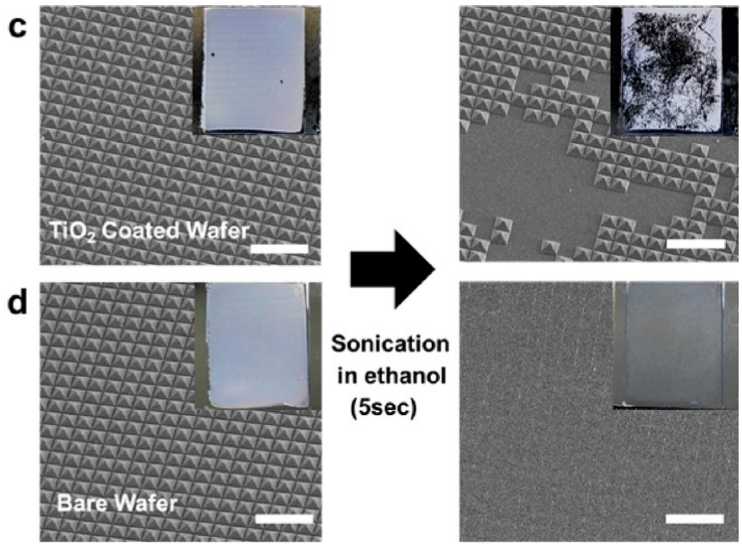

Figure 5. SEM images and photographs on the delamination of isolated microstructures, (prisms (a and b) and pyramids (c and d)) on different substrates (with (a and $\mathrm{c}$ ) and without $(\mathrm{b}$ and $\mathrm{d})$ the $\mathrm{TiO}_{2}$ adhesion promoting layer $\left(\mathrm{TiO}_{2}\right.$ layer thickness: $\sim 100 \mathrm{~nm}$, formed by sintering of $\mathrm{Ti}(\mathrm{IV})$ bis(ethyl acetoacetato) diisopropoxide) coating at $500{ }^{\circ} \mathrm{C}$ for $\left.20 \mathrm{~min}\right)$. SEM images on the left are the isolated microstructures prepared on the substrates, and the SEM images on the right are the same samples after air blowing (please note in $b$ that the prism structures were completely removed upon air blowing) and sonication in ethanol (c, a large portion of pyramids are still left on the substrate, while d, pyramids are completely removed). The scale bars indicate $100 \mu \mathrm{m}$ and the size of wafers in the photographs is $2 \mathrm{~cm} \times 2$ cm.

a Isolated Mesoporous Micro-Patterns (Prisms \& Pyramids)

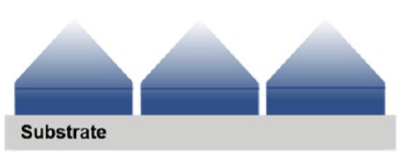
Sonication (for Pyramid)
$\mathrm{N}_{2}$ Blowing (for Prism) or
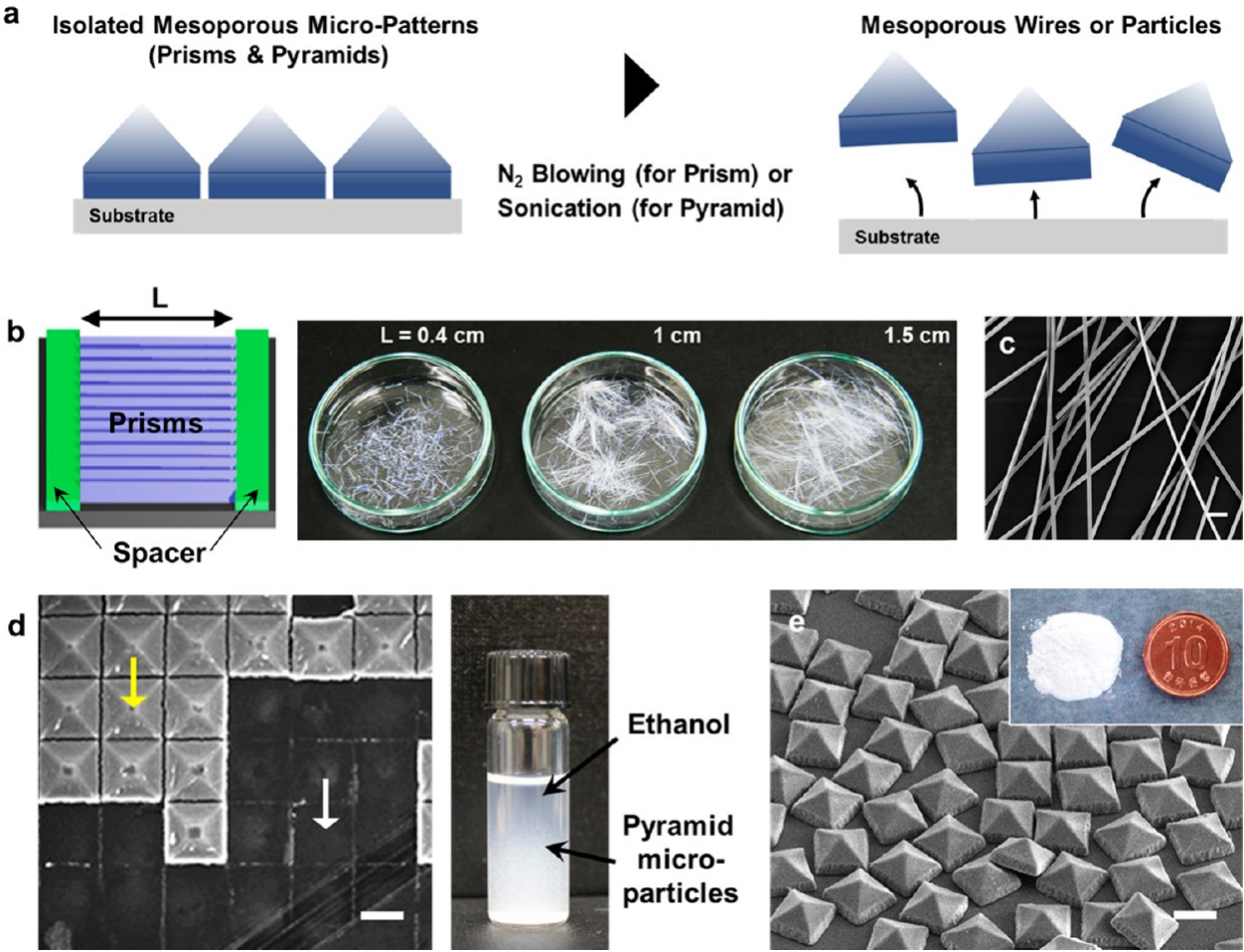

Ethanol

Pyramid microparticles
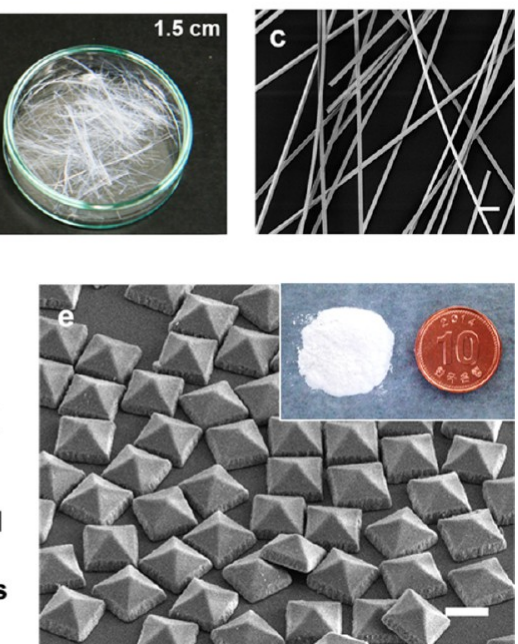

Figure 6. (a) Schematic illustration of the fabrication of mesoporous microstructures (wires and particles). (b) Pictures of collected wires with different length $(0.4,1$, and $1.5 \mathrm{~cm}$ ), which are controlled by the distance between spacers for doctor blade coating. (c) SEM image of mesoporous $\mathrm{TiO}_{2}$ wires completely detached from the substrate by air blowing. (d) SEM image of a footprint of a detached pyramid (white arrow), a remaining isolated pyramid (yellow arrow), and a picture of isolated pyramids dispersed in ethanol. (e) SEM image of collected mesoporous pyramidal $\mathrm{TiO}_{2}$ microparticles. Inset: microparticle powders $(0.3 \mathrm{~g})$ after ethanol removal. The scale bars indicate $100 \mu \mathrm{m}(\mathrm{c})$ and $20 \mu \mathrm{m}$ (d and $\mathrm{e})$, respectively.

Furthermore, heterostructured micropyramid particles incorporating two different materials such as $\mathrm{TiO}_{2}$ and magnetic NPs were successfully prepared by $\mathrm{TiO}_{2}(\sim 20 \mathrm{~nm}) /$ magnetic $\left(\mathrm{Fe}_{2} \mathrm{O}_{3} @ \mathrm{SiO}_{2}\right.$ core-shell) NP mixed pastes. Such heterostructured particles have the photocatalytic effect as well as magnetic properties (Figure $7 \mathrm{e}, \mathrm{f})$. Large surface area $\left(\sim 52 \mathrm{~m}^{2} \mathrm{~g}^{-1}\right.$ measured by BET analysis) originating from mesoporous $\mathrm{TiO}_{2}$ microparticles facilitated the effective photocatalytic reaction, and the magnetic effect originating from $\mathrm{Fe}_{2} \mathrm{O}_{3} @$ $\mathrm{SiO}_{2}$ nanoparticles also enables easy particle collection after photocatalytic reaction (Figure $7 \mathrm{f}$ ). The photocatalytic effect of $\mathrm{TiO}_{2} / \mathrm{Fe}_{2} \mathrm{O}_{3} @ \mathrm{SiO}_{2}$-heterostructured particles was characterized in an aqueous methyl orange dye solution $(0.5 \mathrm{mM}$ in $20 \mathrm{~mL})$. The dyes in the solution were completely decomposed in the presence of $5 \mathrm{mg}$ of the mesoporous $\mathrm{TiO}_{2} /$ magnetic heterostructured particles under UV irradiation $(3.7 \mathrm{~mW} /$ 

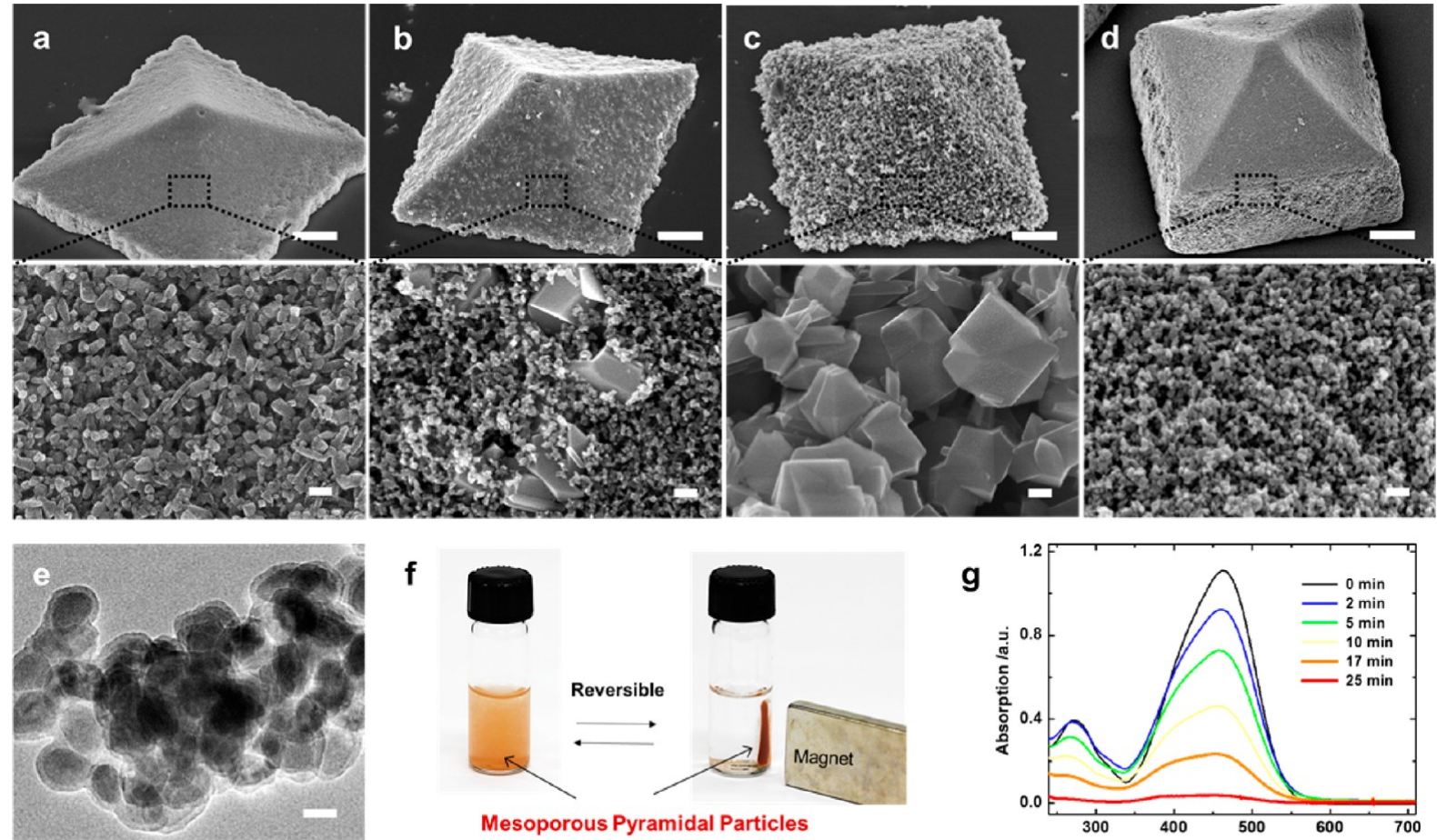

f

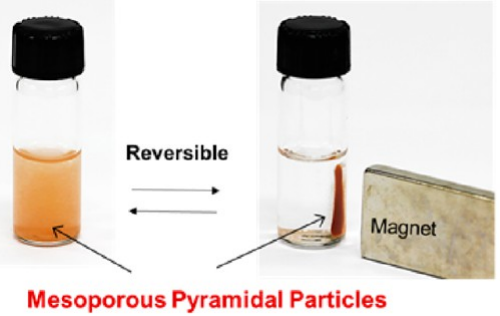

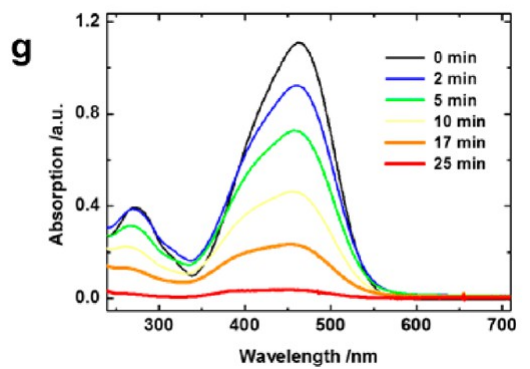

Figure 7. SEM images of mesoporous $\mathrm{TiO}_{2}$ pyramids consisting of (a) $\sim 50 \mathrm{~nm},(\mathrm{~b}) 1: 1$ mixture of $\sim 20$ and $\sim 250 \mathrm{~nm},(\mathrm{c}) \sim 250 \mathrm{~nm} \mathrm{NPs}$, and (d) $\mathrm{a} \mathrm{TiO}_{2}$ pyramid with $\sim 20 \mathrm{~nm}$ sized NPs with a thick residual layer $(\sim 10 \mu \mathrm{m})$. Bottom SEM images are the high magnification images showing $\mathrm{TiO}_{2} \mathrm{NPs}$ used. Scale bars indicate $5 \mu \mathrm{m}$ (top images) and $100 \mathrm{~nm}$ (bottom images), respectively. (e) Transmission electron

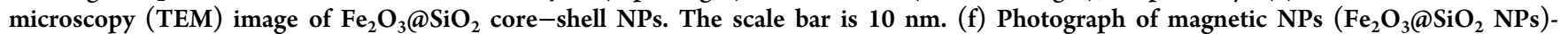
incorporated pyramidal mesoporous $\mathrm{TiO}_{2}$ particles dispersed in water (left) and collected by a magnet (right). (g) UV/vis absorption spectra on the degradation of aqueous methyl orange dye solution mixed with magnetic NP-incorporated mesoporous pyramidal $\mathrm{TiO}_{2}$ particles as a function of UV illumination time.

$\mathrm{cm}^{-2}$ ) in $25 \mathrm{~min}$ (Figure $7 \mathrm{~g}$ ), and after the photocatalytic degradation reaction, the mesoporous microparticles were easily collected by a magnet. This demonstration suggests a reusable and simple photocatalytic reaction process without further cumbersome purification of such photocatalytic particles.

\section{CONCLUSION}

We controlled the cracks on mesoporous microstructures generated by stress localization. The cracks were initiated by soft imprinting with micropatterned (prisms and pyramids) PDMS molds and further propagated by concentrated stress due to the volume shrinkage of the structure upon sintering of $\mathrm{TiO}_{2}$ pastes. We demonstrated that the degree of stress localization was controlled by the thickness of residual layers, NP size, and heating rate. The well-defined cracks propagated along the valleys of patterned microstructures led to separate mesoporous $\mathrm{TiO}_{2}$ microstructures. By detaching those single isolated mesoporous prisms and pyramids from low adhesive substrates, mesoporous $\mathrm{TiO}_{2}$ wires and particles of uniform shape and size were successfully obtained. The feature size and chemical functions of mesoporous wires and particles were easily tuned by the fabrication strategy proposed in the present study. Consequently, precise crack generation and controlled propagation as a result of stress localization with patterned imprints, coupled with delamination from weak adhesive substrates, offer well-defined isolated mesoporous structures with potentials for various applications.

\section{METHODS}

Preparation of Micropatterned PDMS Molds. Prior to soft molding, patterned master structures (prisms and pyramids) were prepared by the micromachining technique. Replica molds were then made with UV-curable polyurethane acrylate (PUA, 301-RM, Minuta Tech) on PET film, and polydimethylsiloxane (PDMS, Sylgard 184 silicon elastomer, Dow Corning) molds were thermally cured out of the PUA molds afterward. ${ }^{30,31}$ The precursor to curing agent ratio of PDMS was chosen to be 10:1 by weight, and the PDMS molds were fully cured at $80{ }^{\circ} \mathrm{C}$ for $8 \mathrm{~h}$.

Soft Molding on $\mathrm{TiO}_{2}$ NP Wet Pastes. The commercial wet pastes containing $20 \mathrm{~nm}$ and $250 \mathrm{~nm} \mathrm{TiO} 2 \mathrm{NPs}$ were purchased from Dyesol (DSL 18NR-T and WER2-O, respectively). The wet NP pastes were uniformly coated on substrates by the doctor blade coating method, and spacers of different thickness were used to control the film thickness. Patterned microstructures were fabricated by soft molding with as-prepared micropatterned PDMS molds, which initially shaped the paste while drying the solvent at $60{ }^{\circ} \mathrm{C}$ for $10 \mathrm{~min}$. After the solvent was dried, the PDMS molds were detached from patterned solidified NP pastes and the molded pastes were subjected to calcination at $500{ }^{\circ} \mathrm{C}$ for $20 \mathrm{~min}$ to decompose all of the remaining organic components. Finally, isolated mesoporous $\mathrm{TiO}_{2}$ microstructures were obtained with controlled cracks formed at the patterned edges in contact with the pastes.

Fabrication of Mesoporous Microwires and -particles. The mesoporous $\mathrm{TiO}_{2}$ microwires and -particles were fabricated from the isolated $\mathrm{TiO}_{2}$ mesoporous microprisms and pyramids on the wafer, respectively. The isolated mesoporous microprisms were detached from the wafer by gentle air blowing and collected as microwires. The micropyramids were also detached from the wafer as microparticles by sonication in ethanol for 3-5 s. To coat a thin additional $\mathrm{TiO}_{2}$ layer that enhanced the mechanical stability of NP networks, the substrates containing micropyramids were dipped in $40 \mathrm{mmol}$ of $\mathrm{TiCl}_{4}$ solution 
at $70{ }^{\circ} \mathrm{C}$ for $30 \mathrm{~min}$ and gently rinsed with deionized water, followed by sintering at $500{ }^{\circ} \mathrm{C}$ for $30 \mathrm{~min}$. The patterned micropyramids were dipped in ethanol and sonicated for $3 \mathrm{~s}$. The micropyramids were easily detached from the substrate and dispersed in ethanol as mesoporous $\mathrm{TiO}_{2}$ microparticles.

Analyses. The scanning electron microscope images were characterized with a field emission scanning electron microscope (FE-SEM, JSM-6701F, JEOL) at an acceleration voltage of $10.0 \mathrm{kV}$. To study the pore characteristics of mesoporous $\mathrm{TiO}_{2}$ structures, a Micromeritics ASAP 2000 system with nitrogen adsorption and desorption was used. The surface area and pore volume were determined from the Brunauer-Emmett-Teller method.

FEM Analysis. FEM analysis of stress distribution was performed using COMSOL Multiphysics software. The behavior of elastic materials was governed by a governing equation of solid mechanics ${ }^{44}$ which was derived by Newton's second law with a strain-displacement relation as follows

$$
\begin{aligned}
& -\sigma_{i j, i}=\rho \ddot{u}_{j} \\
& \varepsilon_{i j}=\frac{1}{2}\left(u_{i, j}+u_{j, i}\right)
\end{aligned}
$$

where $i$ and $j$ indicate the direction of axes $(x, y, z)$ in the Cartesian system, the notation for $X_{i}$ represents the derivative of property $X$ with respect to $i, \rho$ is the mass density of material, $u_{j}$ and $\ddot{u}_{j}$ indicate the components of displacement and acceleration in the $j$-axis, and $\varepsilon_{i j}$ and $\sigma_{i j}$ are the strain and stress in the $i$-direction on the plan whose normal is $j$-axis. In order to describe the volume shrinkage for cracking which was caused by thermal decomposition of organic binders during sintering step, as a load, the pressure, $P_{\mathcal{O}}$ exerted on the upper plane boundary was added. The boundary contacted a PDMS mold directly. $\phi_{\mathrm{NP}}$ is the fraction of NPs in the paste defined as $\phi_{\mathrm{NP}}=V_{\mathrm{NP}} /$ $\left(V_{\text {residual layer }}+V_{\text {structure }}\right)$, where $V_{\mathrm{NP}}$ is the volume of all NPs, $V_{\text {residual layer }}$ $=w l\left(1+\varepsilon_{r}\right) h_{\mathrm{ri}}$ is the volume of residual layer, and $V_{\text {structure }}=\alpha w l(1+$ $\left.\varepsilon_{s}\right) h_{s i}$ is the volume of the structure made by the PDMS mold. $w, l, h_{\mathrm{r} \text {, }}$ $h_{\mathrm{si}}, \varepsilon_{\beta}$, and $\alpha$ are the lateral length, horizontal length, initial height of residual layer, initial height of the structure, strain of $\beta$, and the structure coefficient $(\alpha=1 / 2$ for prism and $\alpha=1 / 3$ for pyramid structure respectively). The strain of $\beta$ is expressed as $\varepsilon_{\beta}=\left(h_{\beta f}-h_{\beta i}\right) /$ $h_{\beta i}$, where $h_{\beta f}$ is the final height of $\beta$. ( $\beta=r$ for the residual layer and $\beta$ $=s$ for the structure). Based on the assumption that NPs are nondeformable materials, when organic binders are fully decomposed, pressure loading is zero at $\phi_{\mathrm{NP}}=1$ because the volume of all the nondeformable NPs cannot be larger than the total volume of residual layer and structure ${ }^{21}$ after the sintering process. The external pressure $P_{\mathrm{c}}$ is introduced to emulate the force caused by the volume shrinkage arising from thermal decomposition of organic materials during sintering step. The $P_{c}$ is defined on the basis of the normalized capillary pressure, ${ }^{45,46}$ which is suitable to examine the effect of remaining organics within interparticle voids on drying behavior, such as consolidation and cracking; the external pressure $P_{c}$ is formed as follows

$$
P_{\mathrm{c}}=C\left(\frac{1-\phi_{\mathrm{NP}}}{\phi_{\mathrm{NP}}}\right)^{n}
$$

where $C$ and $n$ are the arbitrary parameters for fitting $P_{\mathrm{c}}$ according to $\phi_{\mathrm{NP}}$. We chose $C=2.5 \times 10^{5} \mathrm{~N} \mathrm{~m}^{-2}$ and $n=2$ on the basis of experimental data. The external pressure has a rational form of fraction of NPs, $\phi_{\mathrm{NP}}$, as shown in eq 3 , and is exerted on the upper plane of the structure. In addition, the fixed boundary condition $(u=0)$ was set at the bottom planes attached to the substrate. The stress distribution was calculated continuously across the geometric domain by solving eq 1 developed for each of the finite -elements with appropriate boundary conditions and initial conditions. The individual solutions over subdomains are linked together to generate the unified behavior of the entire system, taking into account the continuity at the adjacent elements. ${ }^{47}$

\section{ASSOCIATED CONTENT}

\section{Supporting Information}

The Supporting Information is available free of charge on the ACS Publications website at DOI: 10.1021/acsnano.6b03044.

Figures S1-S7 (PDF)

Movie S1 (AVI)

\section{AUTHOR INFORMATION}

\section{Corresponding Authors}

*E-mail: hsyoon@seoultech.ac.kr.

*E-mail: khchar@plaza.snu.ac.kr.

\section{Author Contributions}

${ }^{\perp}$ S.W., S.L., and Y.L. contributed equally to this work.

\section{Notes}

The authors declare no competing financial interest.

\section{ACKNOWLEDGMENTS}

This work was financially supported by the National Creative Research Initiative Center for Intelligent Hybrids (No. 20100018290) and the IRTG Program (No. 2011-0032203) through a National Research Foundation of Korea (NRF) grant and the BK21 Plus Program funded by the Ministry of Education, Science, and Technology (MEST) of Korea. Additionally, H.Y. acknowledges financial support from the Basic Science Research Program (2013R1A2A2A04015981) funded by the Ministry of Science, ICT, and Future Planning of Korea.

\section{REFERENCES}

(1) Whitesides, G. M.; Bowden, N.; Brittain, S.; Evans, A. G.; Hutchinson, J. W. Spontaneous Formation of Ordered Structures in Thin Films of Metals Supported on an Elastomeric Polymer. Nature 1998, 393, 146-149.

(2) Sun, Y.; Kumar, V.; Adesida, I.; Rogers, J. A. Buckled and Wavy Ribbons of GaAs for High-Performance Electronics on Elastomeric Substrates. Adv. Mater. 2006, 18, 2857-2862.

(3) Kim, P.; Abkarian, M.; Stone, H. A. Hierarchical Folding of Elastic Membranes Under Biaxial Compressive Stress. Nat. Mater. 2011, 10, 952-957.

(4) Singamaneni, S.; McConney, M. E.; Tsukruk, V. V. Spontaneous Self-Folding in Confined Ultrathin Polymer Gels. Adv. Mater. 2010, 22, 1263-1268.

(5) Yang, S.; Khare, K.; Lin, P. C. Harnessing Surface Wrinkle Patterns in Soft Matter. Adv. Funct. Mater. 2010, 20, 2550-2564.

(6) Park, H. G.; Jeong, H. C.; Jung, Y. H.; Seo, D. S. Control of the Wrinkle Structure on Surface-Reformed Poly(dimethylsiloxane) via Ion-Beam Bombardment. Sci. Rep. 2015, 5, 12356.

(7) Juillerat, F.; Bowen, P.; Hofmann, H. Formation and Drying of Colloidal Crystals Using Nanosized Silica Particles. Langmuir 2006, 22, 2249-2257.

(8) Senthilarasu, S.; Peiris, T. A. N.; Garcia-Canadas, J.; Wijayantha, K. G. U. Preparation of Nanocrystalline $\mathrm{TiO}_{2}$ Electrodes for Flexible Dye-Sensitized Solar Cells: Influence of Mechanical Compression. J. Phys. Chem. C 2012, 116, 19053-19061.

(9) Marthelot, J.; Roman, B.; Bico, J.; Teisseire, J.; Dalmas, D.; Melo, F. Self-Replicating Cracks: A Collaborative Fracture Mode in Thin Films. Phys. Rev. Lett. 2014, 113, 085502.

(10) Mahe, M.; Heintz, J. M.; Rodel, J.; Reynders, P. Cracking of Titania Nanocrystalline Coatings. J. Eur. Ceram. Soc. 2008, 28, 20032010.

(11) Choi, S. Y.; Mamak, M.; Coombs, N.; Chopra, N.; Ozin, G. A. Thermally Stable Two-Dimensional Hexagonal Mesoporous Nanocrystalline Anatase, Meso-nc-TiO 2 : Bulk and Crack-Free Thin Film Morphologies. Adv. Funct. Mater. 2004, 14, 335-344. 
(12) Sivaramakrishnan, S.; Chia, P.-J.; Yeo, Y.-C.; Chua, L.-L.; Ho, P. K.-H. Controlled Insulator-to-Metal Transformation in Printable Polymer Composites with Nanometal Clusters. Nat. Mater. 2007, 6, 149-155.

(13) Hatton, B.; Mishchenko, L.; Davis, S.; Sandhage, K. H.; Aizenberg, J. Assembly of Large-Area, Highly Ordered, Crack-Free Inverse Opal Films. Proc. Natl. Acad. Sci. U. S. A. 2010, 107, 1035410359.

(14) Chan, E. P.; Crosby, A. J. Fabricating Microlens Arrays by Surface Wrinkling. Adv. Mater. 2006, 18, 3238-3242.

(15) Chandra, D.; Yang, S.; Lin, P. C. Strain Responsive Concave and Convex Microlens Arrays. Appl. Phys. Lett. 2007, 91, 251912.

(16) Khang, D. Y.; Jiang, H.; Huang, Y.; Rogers, J. A. A Stretchable Form of Single-Crystal Silicon for High-Performance Electronics on Rubber Substrates. Science 2006, 311, 208-212.

(17) Qi, Y.; Jafferis, N. T.; Lyons, K.; Lee, C. M.; Ahmad, H.; McAlpine, M. C. Piezoelectric Ribbons Printed onto Rubber for Flexible Energy Conversion. Nano Lett. 2010, 10, 524-528.

(18) Lipomi, D. J.; Tee, B. C. K.; Vosgueritchian, M.; Bao, Z. Stretchable Organic Solar Cells. Adv. Mater. 2011, 23, 1771-1775.

(19) Kim, J. B.; Kim, P.; Pegard, N. C.; Oh, S. J.; Kagan, C. R.; Fleischer, J. W.; Stone, H. A.; Loo, Y. L. Wrinkles and Deep Folds as Photonic Structures in Photovoltaics. Nat. Photonics 2012, 6, 327-332.

(20) Hutchinson, J. W.; Suo, Z. Mixed Mode Cracking in Layered Materials. Adv. Appl. Mech. 1991, 29, 63-191.

(21) Singh, K. B.; Tirumkudulu, M. S. Cracking in Drying Colloidal Films. Phys. Rev. Lett. 2007, 98, 218302.

(22) Cao, H.; Lan, D.; Wang, Y.; Volinsky, A. A.; Duan, L.; Jiang, H. Fracture of Colloidal Single-Crystal Films Fabricated by Controlled Vertical Drying Deposition. Phys. Rev. E 2010, 82, 031602.

(23) Goehring, L.; Clegg, W. J.; Routh, A. F. Plasticity and Fracture in Drying Colloidal Films. Phys. Rev. Lett. 2013, 110, 024301.

(24) Singh, K. B.; Bhosale, L. R.; Tirumkudulu, M. S. Cracking in Drying Colloidal Films of Flocculated Dispersions. Langmuir 2009, 25, 4284-4287.

(25) Kim, J. Y.; Cho, K.; Ryu, S.; Kim, S. Y.; Weon, B. M. Crack formation and prevention in colloidal drops. Sci. Rep. 2015, 5, 13166.

(26) Livne, A.; Bouchbinder, E.; Svetlizky, I.; Fineberg, J. The Near-

Tip Fields of Fast Cracks. Science 2010, 327, 1359-1363.

(27) Kim, B. C.; Matsuoka, T.; Moraes, C.; Huang, J.; Thouless, M. D.; Takayama, S. Guided Fracture of Films on Soft Substrates to Create Micro/Nano-Feature Arrays with Controlled Periodicity. Sci. Rep. 2013, 3, 3027.

(28) Kang, D.; Pikhitsa, P. V.; Choi, Y. W.; Lee, C.; Shin, S. S.; Piao, L.; Park, B.; Suh, K. Y.; Kim, T. I.; Choi, M. Ultrasensitive Mechanical Crack-Based Sensor Inspired by the Spider Sensory System. Nature 2014, 516, 222-226.

(29) Nam, K. H.; Park, I. H.; Ko, S. H. Patterning by Controlled Cracking. Nature 2012, 485, 221-224.

(30) Wooh, S.; Yoon, H.; Jung, J. H.; Lee, Y. G.; Koh, J. H.; Lee, B.; Kang, Y. S.; Char, K. Efficient Light Harvesting with Micropatterned 3D Pyramidal Photoanodes in Dye-Sensitized Solar Cells. Adv. Mater. 2013, 25, 3111-3116.

(31) Wooh, S.; Koh, J. H.; Lee, S.; Yoon, H.; Char, K. TrilevelStructured Superhydrophobic Pillar Arrays with Tunable Optical Functions. Adv. Funct. Mater. 2014, 24, 5550-5556.

(32) Chiu, R. C.; Garino, T. J.; Cima, M. J. Drying of Granular Ceramic Films: I, Effect of Processing Variables on Cracking Behavior. J. Am. Ceram. Soc. 1993, 76, 2257-2264.

(33) Xu, P.; Mujumdar, A. S.; Yu, B. Drying-Induced Cracks in Thin Film Fabricated from Colloidal Dispersions. Drying Technol. 2009, 27, 636-652.

(34) Boulogne, F.; Giorgiutti-Dauphiné, F.; Pauchard, L. How to Reduce the Crack Density in Drying Colloidal Material? Oil Gas Sci. Technol. 2014, 69, 397-404.

(35) Mukai, S. R.; Nishihara, H.; Shichi, S.; Tamon, H. Preparation of Porous $\mathrm{TiO}_{2}$ Cryogel Fibers through Unidirectional Freezing of Hydrogel Followed by Freeze-Drying. Chem. Mater. 2004, 16, 49874991.
(36) Bao, N.; Li, Y.; Wei, Z.; Yin, G.; Niu, J. Adsorption of Dyes on Hierarchical Mesoporous $\mathrm{TiO}_{2}$ Fibers and Its Enhanced Photocatalytic Properties. J. Phys. Chem. C 2011, 115, 5708-5719.

(37) Dong, X.; Guo, Z.; Song, Y.; Hou, M.; Wang, J.; Wang, Y.; Xia, $\mathrm{Y}$. Flexible and Wire-Shaped Micro-Supercapacitor Based on Ni$(\mathrm{OH})_{2}$-Nanowire and Ordered Mesoporous Carbon Electrodes. Adv. Funct. Mater. 2014, 24, 3405-3412.

(38) Li, H.; Bian, Z.; Zhu, J.; Zhang, D.; Li, G.; Huo, Y.; Li, H.; Lu, Y. Mesoporous Titania Spheres with Tunable Chamber Structure and Enhanced Photocatalytic Activity. J. Am. Chem. Soc. 2007, 129, 84068407.

(39) Li, X.; Gu, M.; Hu, S.; Kennard, R.; Yan, P.; Chen, X.; Wang, C.; Sailor, M. J.; Zhang, J. G.; Liu, J. Mesoporous Silicon Sponge as an Anti-Pulverization Structure for High-Performance Lithium-Ion Battery Anodes. Nat. Commun. 2014, 5, 4105.

(40) Lu, F.; Cai, W.; Zhang, Y. ZnO Hierarchical Micro/ Nanoarchitectures: Solvothermal Synthesis and Structurally Enhanced Photocatalytic Performance. Adv. Funct. Mater. 2008, 18, 1047-1056.

(41) Kim, B. C.; Moraes, C.; Huang, J.; Thouless, M. D.; Takayama, S. Fracture-Based Micro- and Nanofabrication for Biological Applications. Biomater. Sci. 2014, 2, 288-296.

(42) O’Regan, B. C.; Durrant, J. R.; Sommeling, P. M.; Bakker, N. J. Influence of the $\mathrm{TiCl}_{4}$ Treatment on Nanocrystalline $\mathrm{TiO}_{2}$ Films in Dye-Sensitized Solar Cells. 2. Charge Density, Band Edge Shifts, and Quantification of Recombination Losses at Short Circuit. J. Phys. Chem. C 2007, 111, 14001-14010.

(43) Dafinone, M. I.; Feng, G.; Brugarolas, T.; Tettey, K. E.; Lee, D. Mechanical Reinforcement of Nanoparticle Thin Films Using Atomic Layer Deposition. ACS Nano 2011, 5, 5078-5087.

(44) Anandarajah, A. Computational Methods in Elasticity and Plasticity: Solids and Porous Media; Springer: New York, 2010.

(45) Routh, A. F. Drying of Thin Colloidal Films. Rep. Prog. Phys. 2013, 76, 046603-046633.

(46) Salamanca, J. M.; Ciampi, E.; Faux, D. A.; Glover, P. M.; McDonald, P. J.; Routh, A. F.; Peters, A. C. I. A.; Satguru, R.; Keddie, J. L. Lateral Drying in Thick Films of Waterborne Colloidal Particles. Langmuir 2001, 17, 3202-3207.

(47) Chapra, S. C.; Canale, R. P. Numerical Methods for Engineers; McGraw-Hill: New York, 1985. 